\title{
Plataformas digitales más utilizadas durante la actual pandemia (covid-19)
}

Estefanía García Díaz - Universidad de Granada Juan José Padial Suarez - Universidad de Granada Emilio Berrocal de Luna - Universidad de Granada
$0000-0001-6367-2136$

$0000-0003-1960-363 X$

0000-0002-3562-8135

Recepción: 26.06.2021 | Aceptado: 16.10.2021

Correspondencia a través de ORCID: Estefanía García Díaz

0000000163672136

Citar: García Díaz, E, Padial Suárez, JJ y Berrocal de Luna, E (2021) Evaluación de las plataformas digitales más utilizadas durante la actual pandemia (Covid-19). REIDOCREA, 10(30), 21-35.

Resumen: El mundo se encuentra sumergido en una crisis sanitaria debido al COVID-19, de un día para otro la comunidad educativa se vio obligada a cambiar las aulas de nuestros centros por las habitaciones de casa, dónde el proceso de enseñanza se mantiene gracias a las Tecnologías de la Información y la Comunicación (TIC) manifestándose en sus múltiples formas. Esta crisis ha provocado una serie de desafíos en diversas áreas, una de las más afectadas ha sido el campo educativo, trasladándose la modalidad presencial por una modalidad virtual y las plataformas educativas digitales han ocupado un papel importante en esta nueva modalidad. El proceso de enseñanza-aprendizaje mediado por las nuevas tecnologías han provocado una serie de cambios tanto en el profesorado como el propio estudiantado, entre estos cambios se pueden destacar la formación del profesorado y la diferencia de acceso a las nuevas tecnologías por parte del estudiantado. Provocando en ocasiones una fuerte brecha digital y la adaptación de ambos colectivos al nuevo escenario entre otros. Actualmente es difícil imaginar lo que sucederá a partir de esta nueva realidad, pero lo que no se puede negar es el papel cada vez más importante que están representando las TIC en el sistema educativo.

Palabra clave: Plataformas digitales

\section{Most widely used digital platforms in the educational field during the pandemic (covid-19)}

Abstract: The world is immersed in a health crisis due to COVID-19, from one day to the next the educational community was forced to change the classrooms of our centers for the rooms of home, where the teaching process is maintained thanks to the Information and Communication Technologies (ICT) manifesting itself in its multiple forms. This crisis has caused a series of challenges in various areas, one of the most affected has been the educational field, moving the face-to-face modality by a virtual modality and digital educational platforms have occupied an important role in this new modality. The teaching-learning process mediated by new technologies have caused a series of changes both in teachers and students themselves, among these changes can be highlighted teacher training, the difference in access to new technologies by students. Sometimes causing a strong digital divide and the adaptation of both groups to the new scenario among others. It is currently difficult to imagine what will happen from this new reality, but what cannot be denied is the increasingly important role that ICT is playing in the education system.

Keyword: Digital platforms

\section{Introducción}

El mundo se encuentra en un momento de cambio, con ello el surgimiento de una nueva educación que le ha tocado reinventarse y poner en práctica un modelo de enseñanza virtual, la educación se enfrenta a un nuevo reto: ¿Pueden crear las TIC mayores oportunidades no solo para unos cuantos estudiantes sino para todos?

La realidad es que cientos de empresas y centros educativos se han volcado para ofrecer conexión y dispositivos móviles al alumnado en estos momentos, a pesar de esto existe un tema que implica una gran preocupación y, es la cantidad de estudiantes que quedan excluidos del sistema educativo y la falta de habilidades tecnológicas es uno de los principales motivos. 
Según estudios el 93\% de la población tiene acceso a cobertura de banda ancha móvil, pero solo $53 \%$ tiene acceso a Internet debido a la falta de dispositivos o habilidades, esto crea una gran desigualdad y al mismo tiempo una brecha digital cada vez más profunda (Álvarez-Pallete, 2020). La educación y formación ejercen un papel fundamental en la sociedad, por ello la importancia de asegurar una calidad en los procesos y herramientas que se utilizan en la enseñanza, haciendo referencia tanto a contextos formales como no formales. Las plataformas virtuales han adquirido una gran importancia en la actualidad, actuando como herramienta de aprendizaje en el contexto educativo, brindando la capacidad de interacción entre uno o varios usuarios con fines pedagógicos. Vivimos en una sociedad cambiante, rodeados de múltiples variaciones a nivel social, económico, político y tecnológico que provocan el surgimiento de una educación que debe adaptarse a las necesidades de la sociedad, para que esta evolucione al mismo tiempo y se adapte a los nuevos cambios que vayan surgiendo. Tal y como queda reflejado en el marco estratégico de cooperación europea en Educación y Formación 2010-2020 vivimos en la sociedad del conocimiento y del aprendizaje permanente (ET, 2010).

El mundo de la educación y formación se relacionan directamente con el mundo del empleo y del trabajo. Los cambios que están transformando la sociedad hacen que se produzcan nuevas exigencias de cualificación y, por tanto, nuevas necesidades formativas. De ahí, importancia de analizar cómo responde la educación y la formación a estos cambios. Los sistemas educativos están siendo abatidos por dos corrientes, una de ellas centrada en garantizar a los seres humanos una educación de calidad, justa y equitativa. Por otro lado, la del valor de la educación frente a la economía, es decir se busca una adaptación a las demandas del mercado, siguiendo con los objetivos 4 y 8 de desarrollo sostenible de la agenda 2030 se pretende promover el crecimiento económico sostenido, inclusivo y sostenible, el empleo pleno y productivo y el trabajo decente para todos y garantizar una educación inclusiva, equitativa y de calidad y promover oportunidades de aprendizaje durante toda la vida para todos. (ODS, 2015)

Es preciso que la evaluación no se entienda como uniformidad con un único modelo, sino como un proceso en el que se integren diversidad de enfoques metodológicos, técnicas de recogida de datos, análisis de estos mismos y múltiples tomas de decisiones. Dentro de las metodologías aplicadas a los estudiantes de Educación Superior dentro del EESS, se considera la aplicación de plataformas virtuales para apoyar el proceso de enseñanza-aprendizaje del alumnado, docencia y la gestión de los contenidos (Berrocal y Megías, 2015). Ardilla Rodríguez (2011) define que una formación es de calidad cuando potencia en el estudiante el desarrollo de sus capacidades para interactuar e interrelacionarse con docentes y compañeros, y aprender en un ambiente educativo mediado por las tecnologías de la información y de la comunicación. Para evaluar la calidad de las plataformas digitales existen una cantidad de modelos y criterios que se centran en determinados aspectos, dándole cada autor un enfoque distinto.

Torres y Ortega (2003) se centran en tres ámbitos para analizar la calidad y son la técnica, organizativa y creativa, comunicacional y por último didáctica. La calidad técnica hace referencia al conjunto de cualidades que deben de garantizar la solidez y estabilidad de los procesos de gestión y enseñanza aprendizaje:

- Organización del contenido para el adecuado desarrollo de los procesos de Enseñanza-Aprendizaje.

- Canales de comunicación: ofrecen la posibilidad de comunicación sincrónica y asincrónica entre todos los miembros implicados en el proceso educativo. 
- La calidad didáctica: con la incorporación de actividades que permitan integrar de forma coordinada diferentes metodologías apoyadas en los principios de aprendizaje de las teorías conductistas, cognitivistas y constructivistas.

\section{Objetivos o hipótesis}

En el presente estudio se establecen los siguientes objetivos, a través de los cuales se sustenta esta investigación:

- O.G.1. Evaluar las plataformas digitales educativas más utilizadas durante la actual pandemia (Covid-19)

- O.E.1.1 Elaborar un sistema de indicadores de las plataformas digitales educativas.

- O.E.1.2 Analizar las plataformas digitales más utilizadas durante la actual pandemia (Covid-19)

- O.G.2 Identificar las opiniones y percepciones del estudiantado y profesorado sobre las plataformas digitales que están utilizando.

- O.E.2.1. Analizar las opiniones del estudiantado y profesorado a partir de los resultados obtenidos

- O.E.2.2 Analizar los datos obtenidos mediante el cuestionario tras la recogida de la información.

\section{Método}

En función de las características expuestas a lo largo del presente estudio, se ha llevado a cabo una investigación descriptiva de carácter evaluativo abordándolo desde una perspectiva cuantitativa.

Por tanto, la aplicación de dicha metodología permitirá describir de manera cuantitativa las percepciones y opiniones de una población concreta de una forma objetiva y comprobable (Buendía, Colás y Hernández-Pina, 1998)

Gracias al uso de una metodología descriptiva se conocerán las opiniones y percepciones que predominan en la población seleccionada, con el objetivo de conocer con exactitud las opiniones y percepciones que tienen por un lado el profesorado y por otro el estudiantado acerca de las plataformas digitales educativas.

Los pilares básicos en los que se apoya la presente investigación son los siguientes:

- El cuestionario

- El sistema de indicadores

- Estudio de caso

A partir de una detallada revisión bibliográfica, de conceptos básicos y la aplicación de éstos, se obtendrá una visión más clara de la percepción real que tienen actualmente. Esta revisión bibliográfica nos permite dar credibilidad, analizar y contrastar de manera eficaz los contenidos trabajados a lo largo de la presente investigación.

\section{Participantes}

La muestra seleccionada para este estudio se compone de dos estratos: por un lado, estudiantado y profesorado, por otro lado, la selección de las plataformas. 
Para este estudio se ha obtenido una muestra de alumnos/as compuesta por estudiantes que se encuentran en la Universidad o en Educación Secundaria. La muestra del profesorado se compone de profesores/as que ejercen su profesión en Universidad y Educación Secundaria.

Para ello, se estableció contacto con ambas poblaciones mediante un cuestionario, administrado de forma online dadas las circunstancias actuales provocadas por la pandemia.

Para la selección de las plataformas, se realiza una revisión de la literatura y se establecen las plataformas de Moodle, Google Classroom, Zoom, Microsoft Teams y Edmodo.

\section{Instrumentos}

En el presente estudio se han utilizado dos instrumentos para la recogida de los datos, por un lado, dos cuestionarios y por otro un sistema de indicadores.

Para llevar a cabo la realización de este estudio se le proporcionó a la población participante (profesorado y alumnado) un cuestionario Ad Hoc creado para la ocasión, basado en el Cuestionario de evaluación de la calidad de los cursos virtuales de la UNED creado por Santoveña Casal (2010) y el Cuestionario de la plataforma practicum de la Universidad de Granada" creado por Berrocal y Megías (2014).

Este soporte ha sido diseñado con el objetivo de obtener percepciones y opiniones del profesorado y estudiantado acerca de las plataformas digitales. Se presentan una serie de ítems, los cuales se encuentran en una escala de tipo Likert valorado del 1 al 4 , donde 4 es el mayor valor de acuerdo y el 1 menor grado de desacuerdo, también se incluyen preguntas dicotómicas (Sí/No).

\section{Procedimiento}

En primer lugar, se crea a partir de la literatura revisada un sistema de indicadores donde se recogen las dimensiones más importantes de cada una de las plataformas seleccionadas.

En segundo lugar, se tiene en cuenta los cuestionarios mencionados con anterioridad se realiza un cribado de los ítems más relevantes y a partir de este se crea un listado. A partir de esto se obtienen dos cuestionarios uno destinado al profesorado y otro al estudiantado.

En tercer lugar, se procede a administrar los cuestionarios de forma online con el objetivo de llegar al máximo número de personas posibles.

Una vez finalizados los cuestionarios se recabaron los datos y fueron transferidos al software informático SPSS v.25 con la finalidad de analizar los datos obtenidos en los cuestionarios ya cumplimentados, para posteriormente realizar la interpretación y plasmar unos resultados.

Para la creación del sistema de indicadores, se han elaborado unos indicadores de calidad con el fin de clarificar y definir de forma más precisa los ítems que compondrían ambos cuestionarios. De esta forma, y tras realizar el análisis de contenido, se elaboraron los indicadores de calidad por medio de cuatro aspectos básicos presentes en toda plataforma digital como son: finalidad, aspectos técnicos, aspectos didácticos e 
interacción/comunicación. Estos se tomaron como un criterio de medida para posteriormente elaborar ambos cuestionarios.

\section{Análisis de datos}

Tal y como ya se ha expuesto en apartados anteriores, se llevará a cabo un análisis descriptivo con la finalidad de conocer las distintas dimensiones que se tratan en ambos cuestionarios (finalidad, comunicación, aspectos técnicos y didácticos) A continuación, quedan reflejados los resultados obtenidos a lo largo de este estudio, con el objetivo de conocer las percepciones del estudiantado y del profesorado sobre las plataformas digitales que han utilizado durante la actual pandemia. A continuación se presentan imágenes de los ítems más representativos que conforman los cuestionarios, en forma de gráfica

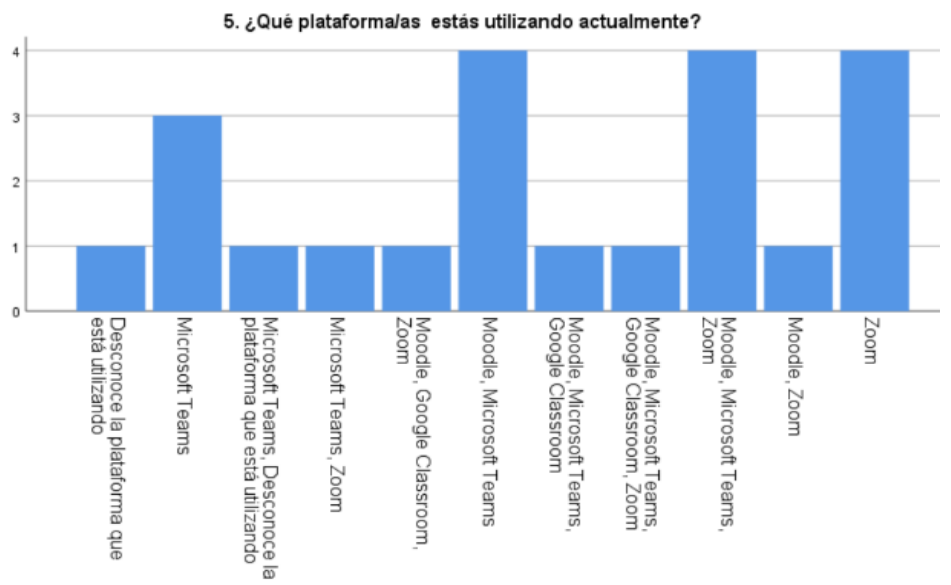

Figura 1. Gráfico de las plataformas que están utilizando actualmente el profesorado

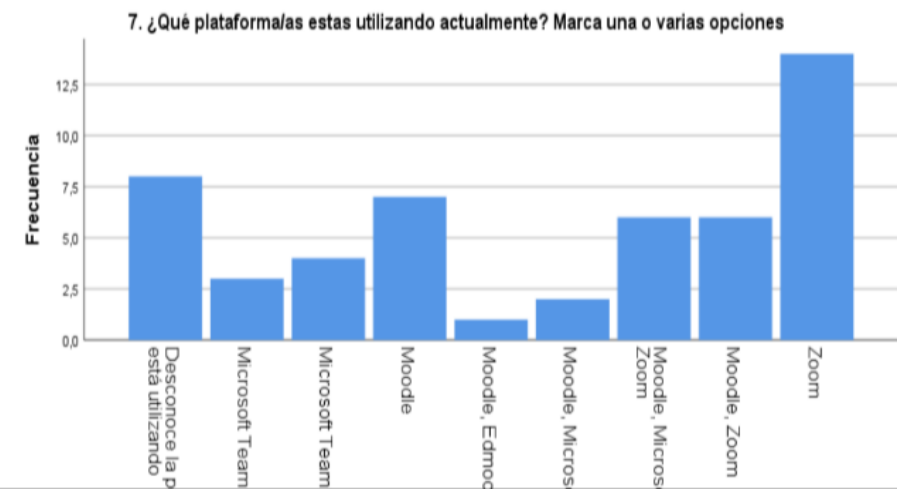

Figura 2 Gráfico de las plataformas que está utilizando actualmente el estudiantado

En los siguientes gráficos se observa que las plataformas más utilizadas por parte del profesorado y estudiantado han sido Moodle, Microsoft Teams y Zoom, coincidiendo ambas muestras. Estas plataformas representan una oportunidad para que las instituciones de enseñanza superior y el profesorado pueda desarrollar de manera específica materiales digitales, cursos online, evaluaciones alternativas como apoyo a 
la enseñanza o continuar con el proceso de enseñanza-aprendizaje cuando no ha sido posible la presencialidad. También se puede observar que una pequeña parte del profesorado y estudiantado desconoce la plataforma que está utilizando.

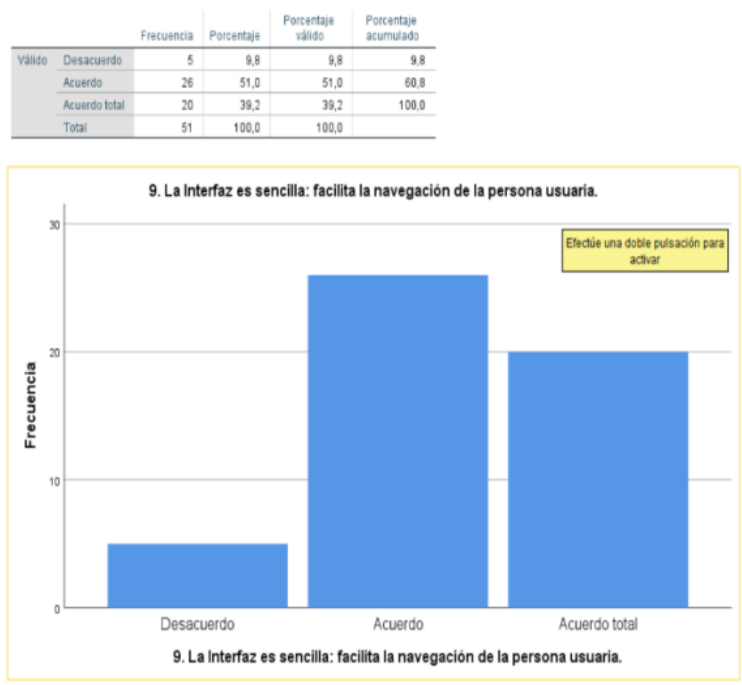

Figura 3. Gráfico perteneciente al ítem "La interfaz es sencilla" perteneciente a la muestra del profesorado

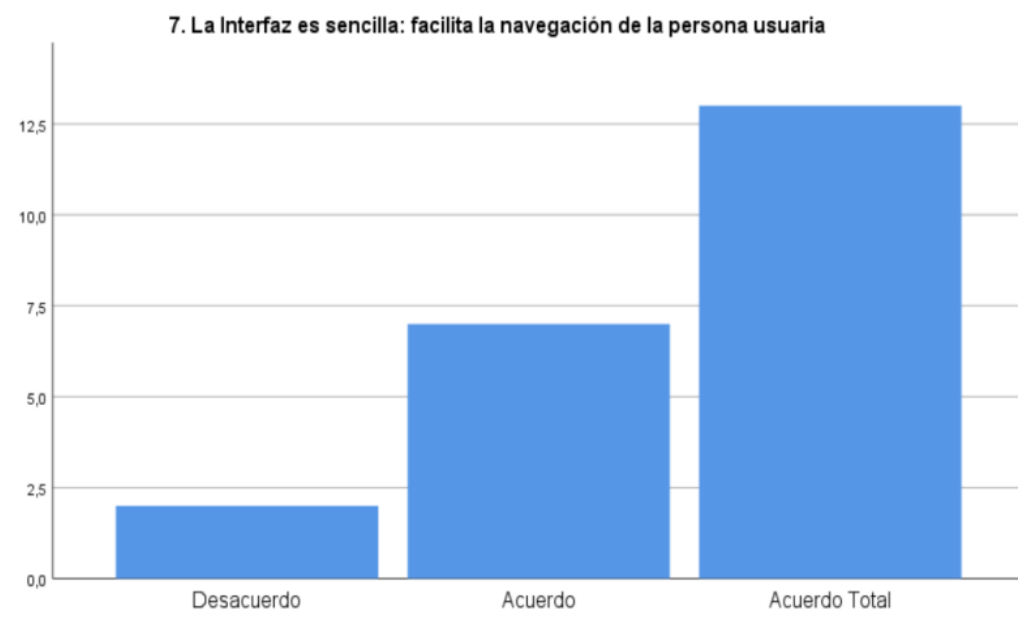

Figura 4. Gráfica perteneciente al ítem "La interfaz es sencilla" correspondiente a la muestra del estudiantado

En esta gráfica se observa que tanto profesorado como estudiantado se sitúa entre el grado de acuerdo y acuerdo total, además la media obtenida en ambos cuestionarios es alta, indicando heterogeneidad en las respuestas obtenidas. Con esto se entiende que la plataforma que están utilizando tiene una interfaz sencilla causando facilidades a la hora de navegar el propio usuario, e incluso facilitándoles la navegación a los usuarios más inexpertos. La interfaz es la que permite que se produzca la interacción entre el ordenador y el usuario. Por ello es importante que su diseño sea sencillo, para que la persona pueda acceder a la información o ejecutar las acciones que desea de una forma simple. Esto permite crear en el estudiante la suficiente confianza para que pueda ubicarse en los distintos apartados y áreas que conforman la plataforma y elaborar un ambiente encaminado al aprovechamiento de los contenidos para conseguir los objetivos previstos. 


\begin{tabular}{|c|c|c|c|c|c|}
\hline & & Frecuencia & Porcentaje & $\begin{array}{c}\text { Porcentaje } \\
\text { válido }\end{array}$ & $\begin{array}{l}\text { Porcentaje } \\
\text { acumulado }\end{array}$ \\
\hline \multirow[t]{4}{*}{ Vâlido } & & 2 & 9,1 & 9,1 & 9,1 \\
\hline & No & 5 & 22,7 & 22,7 & 31,8 \\
\hline & Si & 15 & 68,2 & 68,2 & 100,0 \\
\hline & Total & 22 & 100,0 & 100,0 & \\
\hline
\end{tabular}

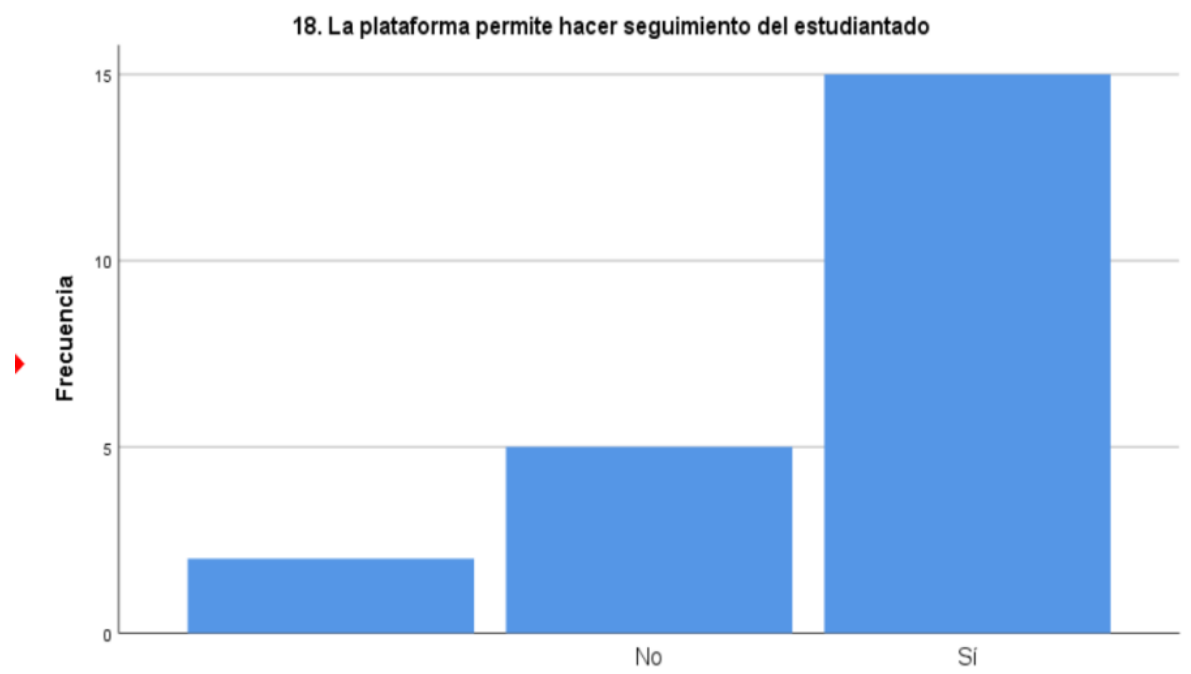

Figura 5. Gráfica perteneciente al ítem "la plataforma permite hacer el seguimiento del estudiantado"

En esta gráfica perteneciente a la muestra del profesorado se puede observar que la mayoría de las respuestas que se obtienen son afirmativas. Esta opción se encuentra en todas las plataformas digitales, ya que es la única opción con la que cuenta el propio docente para conocer los movimientos que realiza el alumnado en la plataforma, el tiempo de conexión, realización de actividades. Haciendo sonar las alarmas en el caso de que haya alumnado que no se conecten o bien no estén entregando las actividades será motivo para que el docente investigue cual puede ser la causa. Dentro de este ítem cabe resaltar la importancia del feedback, autores como Albert Sangra (2001) plantea la retroalimentación como un criterio de calidad en la educación virtual y se considera un elemento clave para que los estudiantes puedan controlar su proceso de enseñanzaaprendizaje.
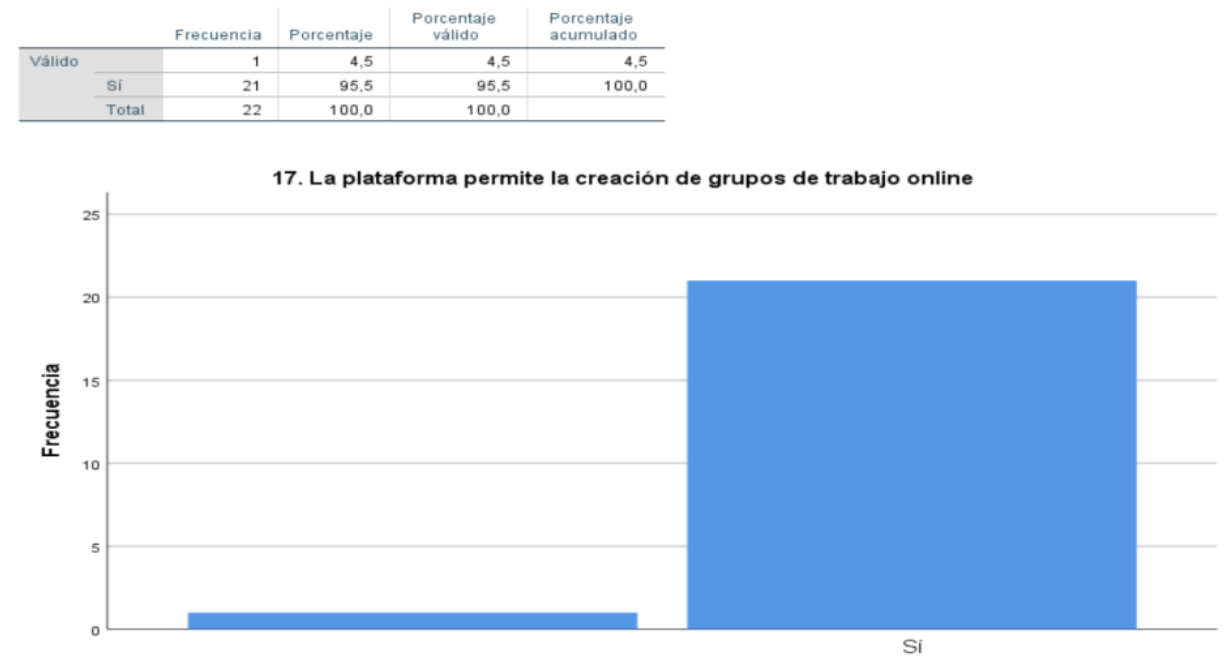

Figura 6 Gráfica que representa el ítem "La plataforma permite la creación de grupos de trabajo online 
En esta gráfica la mayoría de las respuestas que se obtiene por parte del profesorado son afirmativas. Esta opción se incluye en plataformas como Microsoft Teams favoreciendo al profesorado a la hora de realizar una actividad colaborativa creando grupos entre los componentes que se encuentran conectados y estos ser administrados en diversas salas. Teniendo los docentes la posibilidad de visitar cada una de las salas para ver el trabajo que están realizando los grupos en cada una de las salas. En este caso es importante mencionar el papel del aprendizaje colaborativo. El aprendizaje colaborativo se convierte en un componente clave en los procesos educativos a distancia, por eso las facilidades que brinde la plataforma son determinantes para su desarrollo. Hernández y Muñoz (2012) afirman que el aprendizaje colaborativo desarrolla habilidades sociales, preparándose para la vida y el futuro profesional. Slavin (1979) también demuestra que el aprendizaje cooperativo mejora las relaciones entre individuos heterogéneos de distintas etnias y con necesidades educativas especiales, otra razón por la cual es fundamental incluirlo en los procesos de enseñanzaaprendizaje.

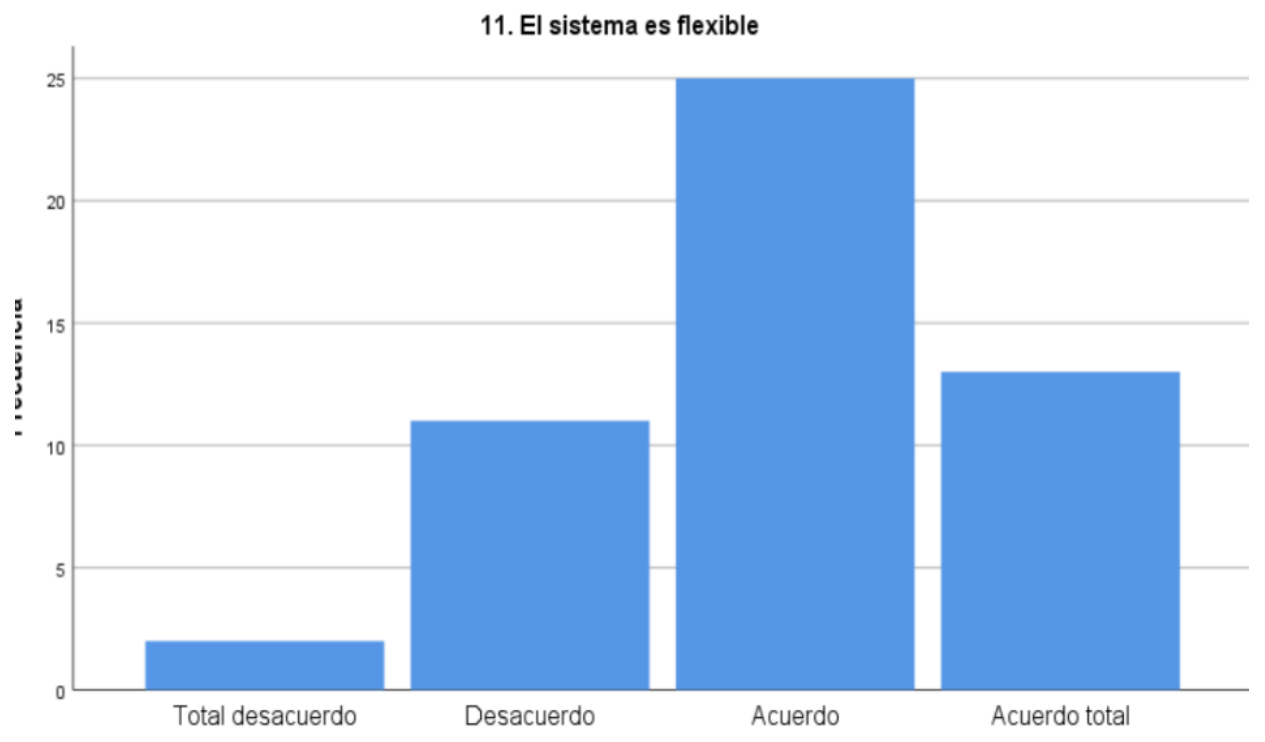

Figura 7 Gráfica perteneciente a la muestra del profesorado

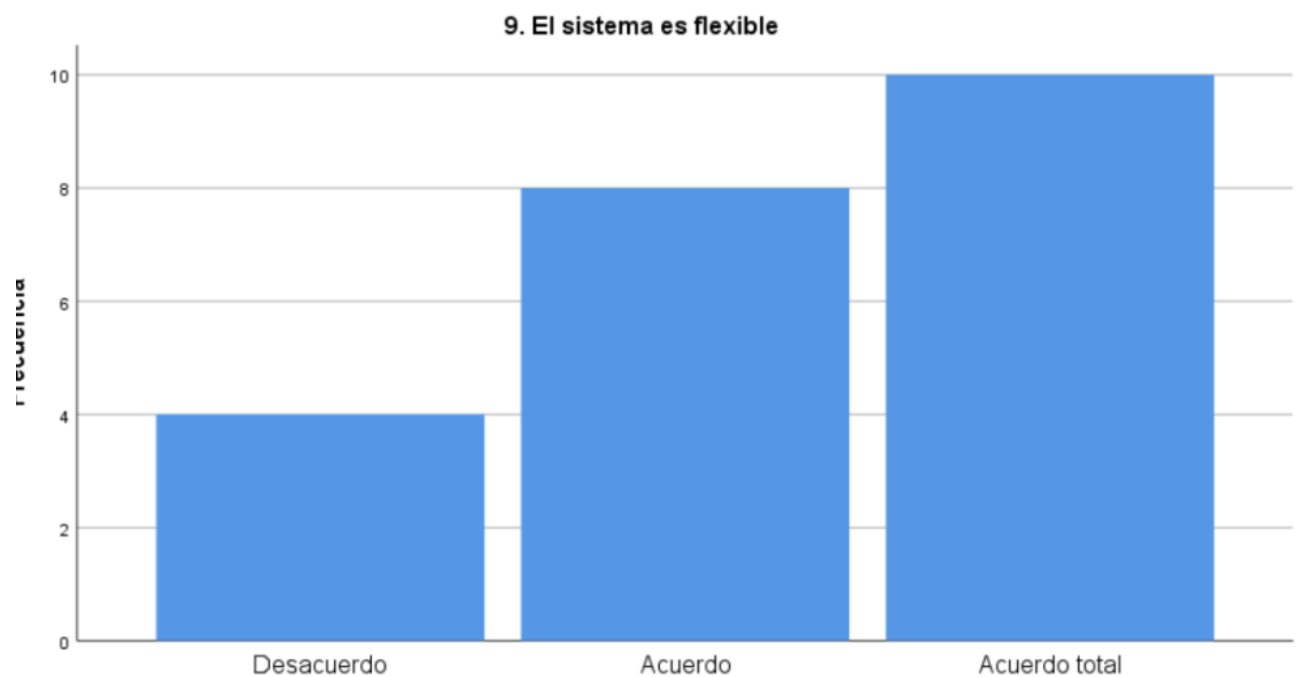

Figura 8 Gráfica perteneciente a la muestra del estudiantado 
Se observa que en la primera gráfica perteneciente a la muestra del profesorado existe heterogeneidad en las respuestas obtenidas, situándose la gran mayoría entre las opciones de acuerdo y acuerdo total. En las respuestas de la muestra del estudiantado se observan respuestas también heterogéneas, aunque también coinciden con la muestra del profesorado situándose en las opciones de acuerdo y acuerdo total. En general se puede observar que existe un alto grado de afirmación respecto a este ítem en ambas muestras. Es importante el usuario pueda acceder sin mayor dificultad desde cualquier dispositivo, por ello es importante que exista compatibilidad con distintos navegadores.

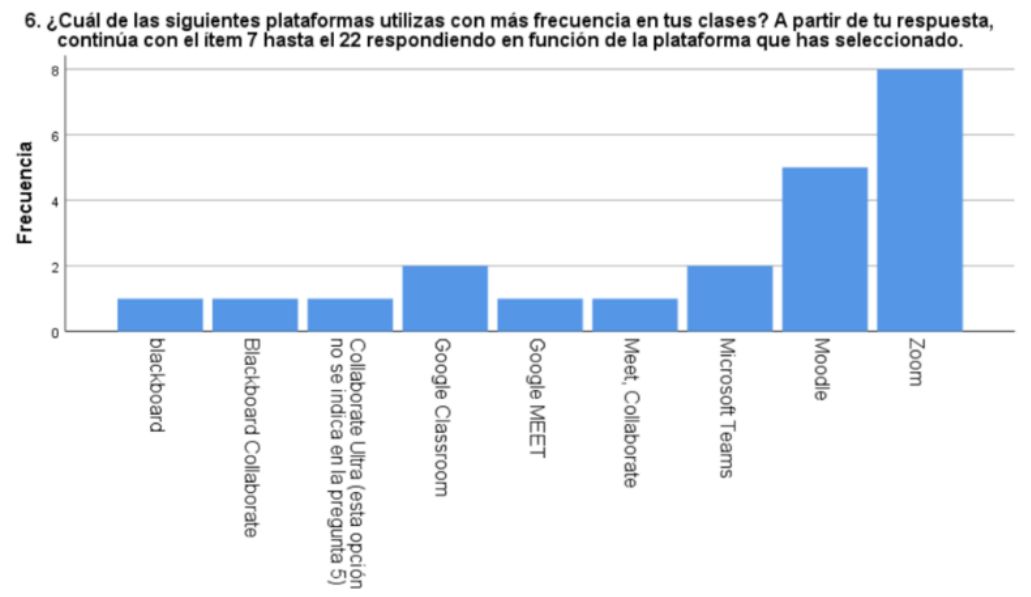

Figura 9 Gráfica que refleja las plataformas más utilizadas por la muestra del profesorado

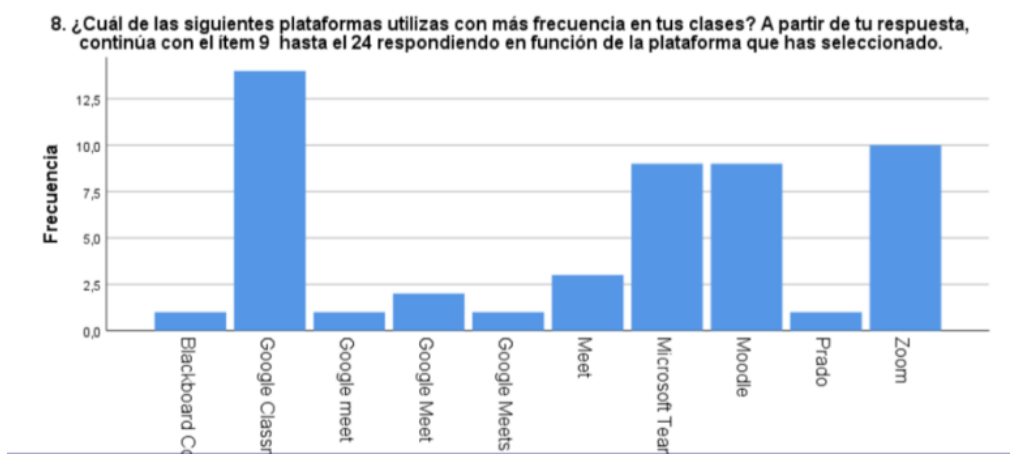

Figura 10 Gráfica que representa las plataformas utilizadas con más frecuencia por el estudiantado

Se observa que en ambos gráficos las plataformas que se han utilizado con más frecuencia han sido Zoom y Google Classroom. Estas plataformas integran herramientas y recursos para administrar, distribuir y controlar contenidos y actividades de formación en línea a través de Internet. También se incluye con bastante frecuencia la plataforma "Blackboard"

Zoom: es una plataforma que permite realizar videoconferencias, chatear e impartir webinars de forma rápida y sencilla. Esta herramienta permite realizar múltiples funciones como por ejemplo realizar reuniones con hasta 100 participantes durante 40 minutos de una forma gratis, es rápida de entender y fácil de usar, lo único que tiene un tiempo determinado para su uso, y no tiene la suficiente seguridad para garantizar la información de los usuarios. Diseñada especialmente para el mundo empresarial, a pesar de todo es utilizada para 48 algunas actividades en la educación, principalmente para videoconferencias. Incluye la opción de compartir pantalla y grabación de la misma, 
pizarrón virtual y calendario. Esta plataforma se ha utilizado con bastante frecuencia no solo en el sector educativo sino también en el sector empresarial. Por otro lado, Google Clasroom, es una plataforma gratuita que permite al personal docente crear aulas virtuales para el intercambio de archivos educativos de texto, de imágenes, de video y de audio con el alumnado. Su función principal consiste en la gestión de un aula de forma colaborativa a través de Internet. Esta plataforma dispone de una aplicación propia para trabajar desde un dispositivo móvil. Es una de las mejores para mejorar el flujo de trabajo de los profesores proporcionándoles un conjunto de herramientas, ideales para utilizarlas con los estudiantes. Además, facilita el aprendizaje colaborativo y el profesorado tiene la opción de ofrecer retroalimentación a través de las actividades (Crawford, 2015)

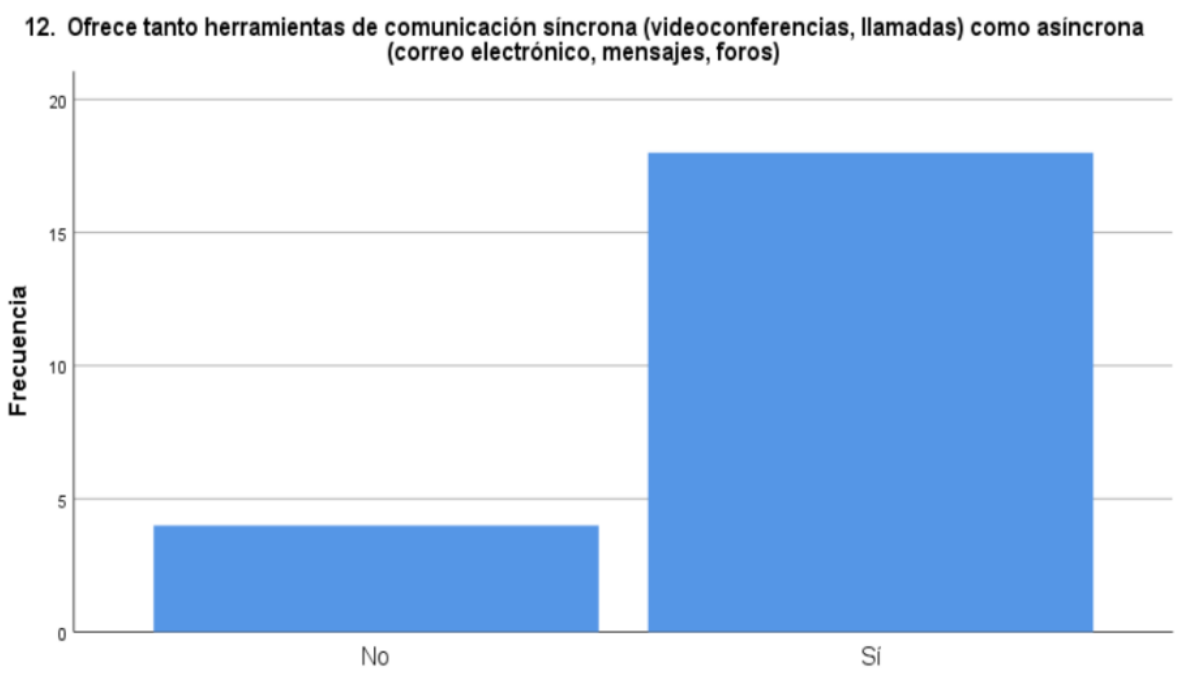

Figura 11 Gráfica que representa el ítem "Ofrece herramientas de comunicación síncrona y asíncrona" perteneciente a la muestra del profesorado.

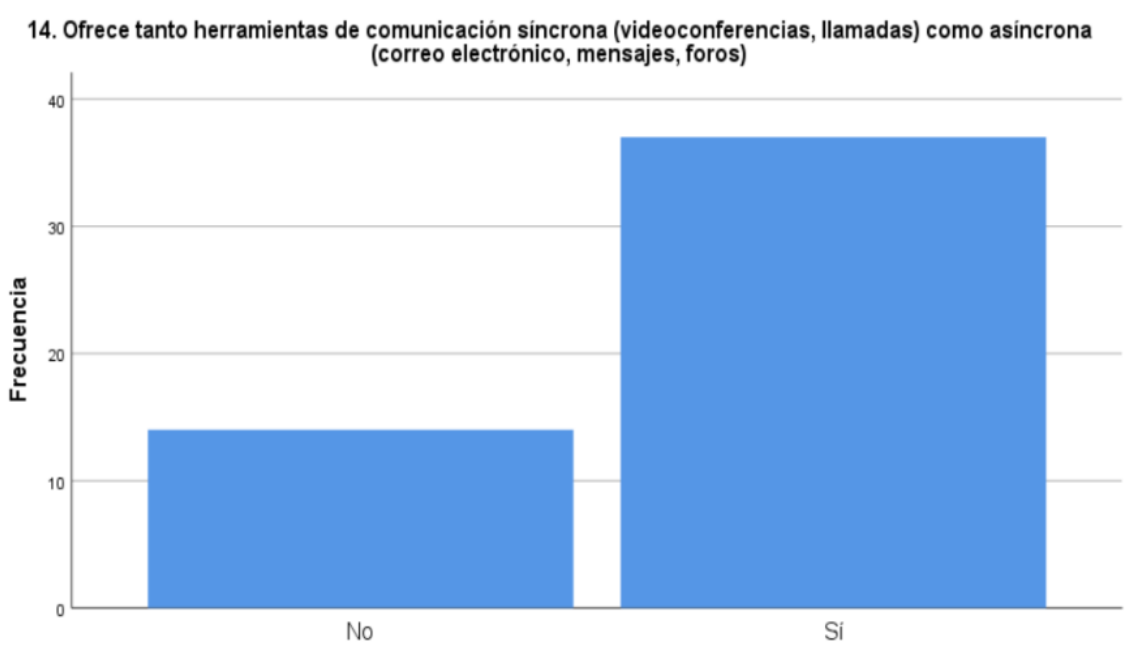

Figura 12 Gráfica que representa el ítem "Ofrece herramientas de comunicación síncrona y asíncrona" perteneciente a la muestra del estudiantado.

En estas gráficas que muestran ambas poblaciones se puede ver que la gran mayoría de los usuarios están de acuerdo con este ítem. Por medio de estas opciones se está fomentando un aprendizaje social, creando una comunidad de estudiantes en línea. Gracias a las plataformas se pueden elaborar grupos de conversación con la presencia del profesorado o sin ella, establecer conversaciones mediante chats, foros, y debates 
donde se compartan las opiniones sobre un mismo tema. Con esto se rompe con el aislamiento que en ocasiones puede provocar el escenario virtual, y el alumnado nunca se sentirá en soledad.

\section{Resultados}

Prestando atención a los resultados obtenidos a lo largo del presente estudio, se destaca la importancia de las plataformas virtuales durante la actual pandemia, donde se ha aumentado el uso de estas en la mayor parte del mundo. No solo en el sector educativo, también en el empresarial, donde a raíz del covid-19 se han continuado con muchos trabajos de forma totalmente online.

Tras la aparición de esta pandemia surgen una serie de nuevos retos a los cuales se ha enfrentado la educación, por un lado, la gran brecha digital y social que se abre. Tal y como refleja Fernández Enguita (2020) la situación de urgencia provocada por esta pandemia ha puesto de manifiesto la existencia de tres brechas principales que se destacan a continuación:

1. Una brecha de acceso, relacionada con tener o no tener acceso a dispositivos electrónicos y/o a conexión a Internet.

2. Una brecha de uso, relacionada con el tiempo de uso y la calidad de este, porque habrá hogares que sí cuentan con dispositivos, pero se comparten entre los miembros de la familia.

3. Una brecha de competencias, relacionada con las competencias digitales del profesorado y del estudiantado para utilizar adecuadamente las plataformas digitales con fines educativos y la capacidad de crear o proveer contenidos y actividades educativas a través de estas.

Con el estallido de la pandemia también surge la necesidad de formación en las nuevas tecnologías, es cierto que las plataformas que se mencionan con anterioridad no necesitan una formación específica para poder utilizarlas, pero si requieren unos conocimientos informáticos mínimos. El sistema educativo ha conseguido que tanto profesorado como alumnado tengan unos conocimientos mínimos para poder adaptarse a esta nueva modalidad, por medio de píldoras formativas, talleres, vídeos. Todo esto con el objetivo de continuar con el proceso de enseñanza-aprendizaje, cambiando el escenario presencial por el virtual.

El uso de estas plataformas proporciona una serie de ventajas entre las que se pueden destacar el empleo de herramientas de comunicación tanto síncronas como asíncronas. La mayoría de las plataformas digitales ofrecen ambas opciones tal y como se puede ver reflejado en los gráficos anteriores. Ya que la comunicación se convierte en un elemento esencial en los procesos de enseñanza aprendizaje y aún más en la modalidad online.

\section{Discusión}

La actual pandemia (Covid-19) ha causado una serie de cambios en varios factores que influyen en el desarrollo de la vida de las personas, siendo uno de los más afectados el educativo, ya que se cerraron de forma inmediata todas las instituciones y centros educativos de cualquier parte del mundo. En cuestión de días se tuvo que cambiar de mentalidad, pasando todo lo programado para realizarlo en un escenario presencial a una modalidad totalmente online y como consecuencia una serie de problemas a los que el sector educativo debía de hacer frente. Hay que destacar la formación del profesorado, recursos disponibles de cada familia, brecha digital y social (Dressen et al., 
2020). Esta pandemia también nos ha mostrado la importancia de incluir las competencias digitales en el mundo de la educación, así como el manejo básico de determinadas plataformas, no solo del alumnado sino también del profesorado. Ya que la capacidad de dominar las nuevas tecnologías se convierte en una necesidad para estos, permitiéndoles seleccionar y conocer las herramientas que mejor se adapten a sus estudiantes para conseguir cubrir las competencias y conseguir los objetivos previstos de cada curso (Monroy, 2020). Las plataformas digitales permiten hacer el seguimiento del alumnado, proporcionándoles al profesorado información detallada de cada uno de los estudiantes, como por ejemplo cuando fue la última vez y primera vez de acceso a la plataforma, cuánto tiempo ha permanecido conectado, incluso el progreso a lo largo de todo el tema, observar los envíos de tareas y conocer si lleva al día los contenidos que se están tratando en los temarios. Esta opción permite al profesorado detectar al alumnado que se encuentra en situación de riesgo de "abandono" y tomar las medidas que sean necesarias para evitar que se den situaciones de este tipo para que ningún estudiante se quede atrás en el proceso de enseñanzaaprendizaje. Por otro lado, plataformas como Google Meet ofrecen la opción de crear grupos de trabajo dividiendo a todos los estudiantes en diferentes salas (breakout rooms) favoreciendo el trabajo colaborativo. (Aguilar et al., 2019)

Como se ha podido observar a lo largo de este estudio las plataformas más utilizadas por profesorado y estudiantado han sido Zoom, Moodle, Microsoft Teams y Google Classroom. Gracias a estas ha sido posible desarrollar actividades interactivas que han facilitado los procesos educativos a través de acciones como la creación de grupos para trabajar una determinada temática, tutorías virtuales, utilizar el vídeo y fotografías para interaccionar y mantener el papel activo del alumnado.

\section{Referencias}

Aguilar, M. E. U., Minnaard, V. A., Rabino, M. C., Minnaard, C. L., \& Comoglio, M. S. (2019, January). Uso de plataformas virtuales para el desarrollo de trabajo colaborativo. Congreso Internacional de Educación y Aprendizaje

Álvarez-Pallete, J.M (2020) Aprender, Enseñar y Trabajar después del 2020. EnlightED Virtual Edition.

Ardila-Rodríguez, M. (2011). Indicadores de calidad de las plataformas educativas digitales. Educación y educadores, 14(1), 189-206.

Berrocal, E., y Megías, S. (2015). Indicadores de calidad para la evaluación de plataformas virtuales. Textos. Revista Internacional de aprendizaje y cibersociedad, 152-172.

Buendía Eisman, L., Colás Bravo, M., y Hernández Pina, F. (1998). Métodos de investigación en psicopedagogía.

Casal, S. M. S. (2010). Cuestionario de evaluación de la calidad de los cursos virtuales de la UNED. Revista de Educación a Distancia (RED), (25).

Crawford, M. (2015). Connecting leadership, professional development and affect. En P. Burnard, B. M. Apelgren y N. Cabaroglu (Ed.), Transformative teacher research: Theory and practice for the C21st.. Boston: Sense Publishers.

Enguita, M. F. (2020). El virus, ese gran innovador. Cuadernos de pedagogía, (512), 154-161. http://reddigital.cnice.mec.es/6/Documentos/docs/articulo13 material.pdf

Monroy, G. (2020). Herramientas tecnológicas aplicadas a la educación a distancia, https://www.gicesperu.org/articulo.php?id=q+sNp2eAe7ON4EYpqsMuAQ

Sangra, A (2001) La calidad de las experiencias virtuales de Educación Superior.

Torres Toro, S. y Ortega Carrillo, S. (2003), Indicadores de calidad en plataformas de formación virtual: una aproximación sistemática, http://www.ugr. es/ sevimeco/revistaeticanet/Cultura eval/Calidad en el eLearning.doc 
Anexos

Anexo 1: Tabla de Indicadores

\begin{tabular}{|c|c|c|c|c|}
\hline PLATAFORMA & FINALIDAD & ASPECTOS TÉCNICOS & COMUNICACIÓN/INTERACCIÓN & $\begin{array}{l}\text { ASPECTOS } \\
\text { DIDÁCTICOS }\end{array}$ \\
\hline $\begin{array}{l}\text { GOOGLE } \\
\text { CLASSROOM }\end{array}$ & $\begin{array}{l}\text {-Creación de } \\
\text { actividades } \\
\text {-Mejora del flujo de } \\
\text { trabajo de profesores } \\
\text {-Facilita Aprendizaje } \\
\text { colaborativo }\end{array}$ & $\begin{array}{l}\text {-Gratuita } \\
\text {-Diseño sencillo } \\
\text {-No modificable } \\
\text { Limpieza y simplicidad en } \\
\text { la interfaz } \\
\text {-Calendario } \\
\text {-Aplicación propia }\end{array}$ & $\begin{array}{l}\text {-Herramientas de Comunicación } \\
\text { Asíncrona (correo electrónico, } \\
\text { Tablón) } \\
\text {-Herramientas de Comunicación } \\
\text { Síncrona (videoconferencia a } \\
\text { través de google meet) }\end{array}$ & $\begin{array}{l}\text {-E-Learning y B-Learning } \\
\text {-Asignación de tareas de } \\
\text { forma selectiva (atención } \\
\text { a la diversidad) } \\
\text {-Evaluación (rúbricas, } \\
\text { múltiples opciones, } \\
\text { kahoot) }\end{array}$ \\
\hline MOODLE & $\begin{array}{l}\text {-Crear espacios de } \\
\text { enseñanza online } \\
\text {-Fomento aprendizaje } \\
\text { colaborativo } \\
\text {-Aprendizaje } \\
\text { constructivista }\end{array}$ & $\begin{array}{l}\text {-Interfaz compleja } \\
\text { (necesidad de } \\
\text { conocimientos de } \\
\text { programación) } \\
\text {-Flexibilidad } \\
\text {-Software libre } \\
\text {-Organización modular }\end{array}$ & $\begin{array}{l}\text {-Herramientas de comunicación } \\
\text { asíncrona (Foro, mensajes, } \\
\text { correos) }\end{array}$ & $\begin{array}{l}\text {-Evaluación (variedad de } \\
\text { cuestionarios, feedback) } \\
\text {-B-learning y E-learning }\end{array}$ \\
\hline ZOOM & $\begin{array}{l}\text {-Comunicación entre } \\
\text { usuarios } \\
\text {-Realizar } \\
\text { videoconferencias, } \\
\text { chatear e impartir } \\
\text { webinars }\end{array}$ & $\begin{array}{l}\text {-Compatibilidad con otros } \\
\text { dispositivos } \\
\text {-Interfaces visuales con } \\
\text { contraste de color y } \\
\text { tamaño para personas con } \\
\text { necesidades específicas } \\
\text { de visión } \\
\text {-Posibilidad de acceso } \\
\text { desde el teclado }\end{array}$ & $\begin{array}{l}\text {-Herramientas de comunicación } \\
\text { Síncrona (videoconferencias) } \\
\text {-Herramientas de comunicación } \\
\text { Asíncrona (chat) }\end{array}$ & $\begin{array}{l}\text {-Evaluación (entrevista } \\
\text { oral) } \\
\text {-Componer grupos de } \\
\text { trabajo } \\
\text {-Grabación de la pantalla } \\
\text {-Envío de archivos } \\
\text { durante la clase }\end{array}$ \\
\hline $\begin{array}{l}\text { MICROSOFT } \\
\text { TEAMS }\end{array}$ & $\begin{array}{l}\text {-Establecer } \\
\text { comunicación y } \\
\text { coordinación entre } \\
\text { alumnado y } \\
\text { profesorado } \\
\text {-Compartir información }\end{array}$ & $\begin{array}{l}\text {-Personalizable } \\
\text {-Gratuita } \\
\text {-Calendario }\end{array}$ & $\begin{array}{l}\text {-Herramientas de Comunicación } \\
\text { Síncrona (videoconferencias, } \\
\text { llamada de audio) } \\
\text {-Herramientas de Comunicación } \\
\text { Asíncrona (Chat, } \\
\text { publicaciones/tablón) }\end{array}$ & $\begin{array}{l}\text {-Programación de } \\
\text { reuniones } \\
\text {-Compartir información } \\
\text { variada } \\
\text {-Equipos } \\
\text {-Evaluación } \\
\text { personalizada } \\
\text {-B-Learnig y E-Learning }\end{array}$ \\
\hline EDMODO & $\begin{array}{l}\text {-Fomento del } \\
\text { aprendizaje } \\
\text { colaborativo } \\
\text {-Aprendizaje } \\
\text { constructivista } \\
\text {-Comunicación entre } \\
\text { docentes y alumnado }\end{array}$ & $\begin{array}{l}\text {-Software privado } \\
\text {-Personalización de } \\
\text { paneles } \\
\text {-Interfaz sencilla } \\
\text {-Interfaz propia para } \\
\text { familias }\end{array}$ & $\begin{array}{l}\text {-Herramientas de comunicación } \\
\text { asíncrona (muro grupal/tablón, } \\
\text { comunidad de aprendizaje, } \\
\text { mensajes) }\end{array}$ & $\begin{array}{l}\text {-Actividades que } \\
\text { incluyen contenido } \\
\text { multimedia variado } \\
\text {-Calendario y libro de } \\
\text { calificaciones } \\
\text {-Evaluación (4 } \\
\text { opciones de } \\
\text { cuestionarios, feedback) } \\
\text {-Biblioteca (compartir } \\
\text { recursos/contenidos) } \\
\text {-Sistemas de Insignias }\end{array}$ \\
\hline
\end{tabular}


1. Tipo de Institución educativa a la que pertenece: Universidad/Educación Secundaria

2. Titulación actual:

3. Curso en el que se encuentra actualmente matriculado:

4. Edad

17 o menor

$18-25$

26-30

Más de 30

5. Sexo

Hombre

Mujer

Prefiero no decirlo

6. ¿Cuándo has utilizado plataformas educativas virtuales?

Antes de la pandemia

Después de la pandemia

Ambas opciones

7. ¿Qué plataformas estas utilizando actualmente? Marca una o varias opciones

Moodle

Microsoft Teams

Zoom

Edmodo

Desconoce la plataforma que está utilizando

8. ¿Cuál de las siguientes plataformas utilizas con más frecuencia en tus clases? A partir de tu respuesta, continúa con el ítem 9 hasta el

25 respondiendo en función de la plataforma que has seleccionado

Moodle

Microsoft Teams

Google Classroom

Zoom

Edmodo

Otro:

9. La interfaz es sencilla: facilita la navegación de la persona usuaria: 1-2-3-4

10. Incluye calendario para una mejor organización

Sí

No

11. El sistema es flexible: 1-2-3-4

12. Se requiere una formación específica para el manejo de la plataforma

Sí

No

13. La plataforma ofrece la posibilidad además de realizar actividades online, actividades presenciales

Sí

No

14. Ofrece tanto herramientas de comunicación síncrona (videoconferencias, llamadas) como de comunicación asíncrona (correo electrónico, mensajes, foros)

Sí

No

15. El sistema es fácil de utilizar: 1-2-3-4

16. El uso de las plataformas digitales te ha facilitado la continuidad en el proceso enseñanza-aprendizaje: 1-2-3-4

17. La plataforma únicamente se utiliza para la educación en línea

Sí

No

18. Contiene una aplicación móvil propia para teléfonos inteligentes, móviles o tabletas

Sí

No

19. Existe un enlace para emitir sugerencias o incidencias a la persona que sea pertinente, si surge algún problema, de forma clara y rápida

Sí

No

20. El uso de las plataformas digitales te ha facilitado la continuidad en el proceso enseñanza-aprendizaje: $1-2-3-4$

21. Permite la personalización adaptándose a las necesidades (físicas, funcionales, etc.) de la persona usuaria: 1-2-3-4

22. La plataforma muestra las actualizaciones

- Sí

No

23. La plataforma muestra las actualizaciones de forma clara

Sí

No

24. Favorece la comunicación entre compañeros mediante herramientas del tipo chats, foros, debates, etc.: $1-2-3-4$

25. Fomenta el trabajo colaborativo: $1-2-3-4$

\section{Anexo 3: Cuestionario del profesorado}

1. ¿Cuántos años de experiencia docente tiene? 
0-5 años

5-10 años

Más de 15 años

2. Tipo de Institución Educativa a la que pertenece

Universidad

Enseñanza Secundaria

3. Sexo

Hombre

Mujer

Prefiero no decirlo

4. Edad

30 o menor

$31-40$

$41-55$

Más de 56

5. ¿Qué plataformas estas utilizando actualmente? Marca una o varias opciones

Moodle

Microsoft Teams

Zoom

Edmodo

Desconoce la plataforma que está utilizando

6. ¿Cuál de las siguientes plataformas utilizas con más frecuencia en tus clases? A partir de tu respuesta, continúa con el ítem 9 hasta el

25 respondiendo en función de la plataforma que has seleccionado

Moodle

Microsoft Teams

Google Classroom

Zoom

Edmodo

Otro:

7. La interfaz es sencilla: facilita la navegación de la persona usuaria: 1-2-3-4

8. Incluye calendario para una mejor organización

Sí

No

9. El sistema es flexible: $1-2-3-4$

10. Se requiere una formación específica para el manejo de la plataforma

Sí

No

11. La plataforma ofrece la posibilidad además de realizar actividades online, actividades presenciales

Sí

No

12. Ofrece tanto herramientas de comunicación síncrona (videoconferencias, llamadas) como de comunicación asíncrona (correo electrónico, mensajes, foros)

$\square$ Sí

ㄱo

13. El sistema es fácil de utilizar: 1-2-3-4

14. El uso de las plataformas digitales te ha facilitado la continuidad en el proceso enseñanza-aprendizaje: 1-2-3-4

15. La plataforma únicamente se utiliza para la educación en línea

$\square$ Sí

No

16. Contiene una aplicación móvil propia para teléfonos inteligentes, móviles o tabletas

- Sí

ㄱo

17. La plataforma permite la creación de grupos de trabajo online

$\square$ Sí

No

18. La plataforma permite hacer seguimiento del alumnado

Sí

No

19. La plataforma es capaz de soportar múltiples conexiones permitiendo trabajar de forma rápida y eficaz

Sí

No

20. La plataforma ofrece seleccionar diferentes tipos de actividades que permite desarrollar la motivación y el papel activo del estudiantado: 1-2-

3-4

21. Las familias del estudiantado tienen una interfaz propia de acceso a la plataforma para estar informados del avance de sus hijos e hijas

Sí

No

22. La plataforma permite la grabación de videoconferencias: 1-2-3-4 